\title{
On 0-controllability and pursuit problems for linear discrete systems under total constraints on controls
}

\begin{abstract}
We consider linear discrete control and pursuit game problems. Control vectors are subjected to total constraints, which are discrete analogues of the integral constraint. By definition, (i) the control system is 0-controllable on the whole if there is a control such that the state of the system $\mathrm{z}(\mathrm{t}) \mathbb{A}[\mathbf{0}$ at some step $\mathrm{t}$, (ii) pursuit can be completed if there exists a strategy of the pursuer such that for any strategy of the evader the state of the system $y(t)$ (m) sufficient condition for equivalence of 0-controllability and completion of the game from any initial position of the space.
\end{abstract}

Keyword: Linear discrete systems; Pursuit game problems; Linear transformation 